\title{
Monotone convolution semigroups
}

by

TAKahiro Hasebe (Kyoto)

\begin{abstract}
We study how a property of a monotone convolution semigroup changes with respect to the time parameter. Especially we focus on "time-independent properties": in the classical case, there are many properties of convolution semigroups (or Lévy processes) which are determined at an instant, and moreover, such properties are often characterized by the drift term and Lévy measure. In this paper we exhibit such properties of monotone convolution semigroups; an example is the concentration of the support of a probability measure on the positive real line. Most of them are characterized by the same conditions on drift terms and Lévy measures as known in probability theory. These kinds of properties are mapped bijectively by a monotone analogue of the Bercovici-Pata bijection. Finally we compare such properties with classical, free, and Boolean cases, which will be important in an approach to unify these notions of independence.
\end{abstract}

1. Introduction. Muraki defined a monotone convolution as the probability distribution of the sum of two monotone independent random variables [15, 16. Let $G_{\mu}(z)(z \in \mathbb{C} \backslash \mathbb{R})$ be the Cauchy transform of a probability measure $\mu$ and $H_{\mu}(z)$ be the reciprocal of $G_{\mu}(z)$. Then $H_{\mu}$ is analytic and maps the upper half plane into itself. Moreover, $\inf _{\operatorname{Im} z>0} \operatorname{Im} H_{\mu}(z) / \operatorname{Im} z=1$. Consequently, $H_{\mu}(z)$ can be expressed uniquely in the form

$$
H_{\mu}(z)=z+b+\int_{\mathbb{R}} \frac{1+x z}{x-z} \eta(d x)
$$

where $b \in \mathbb{R}$ and $\eta$ is a positive finite measure (see [1]). The monotone convolution $\mu \triangleright \nu$ of probability measures $\mu$ and $\nu$ is characterized by

$$
H_{\mu \triangleright \nu}(z)=H_{\mu}\left(H_{\nu}(z)\right) \text {. }
$$

Using this characterization, Muraki classified monotone (or $\triangleright$ - for short) infinitely divisible distributions with compact supports. The complete classification including probability measures with unbounded supports was given by Belinschi [4], as follows.

2010 Mathematics Subject Classification: Primary 46L53, 46L54; Secondary 34A25.

Key words and phrases: monotone convolution, monotone independence, infinitely divisible distributions, Lévy-Khinchin formula. 
THEOREM 1.1. There is a one-to-one correspondence among the following four objects:

(1) $a \triangleright$-infinitely divisible distribution $\mu$;

(2) a weakly continuous monotone convolution semigroup $\left\{\mu_{t}\right\}$ with $\mu_{0}=\delta_{0}, \mu_{1}=\mu$

(3) a composition semigroup of reciprocal Cauchy transforms $\left\{H_{t}\right\}_{t \geq 0}$ $\left(H_{t} \circ H_{s}=H_{t+s}\right)$ with $H_{0}=\mathrm{id}, H_{1}=H_{\mu}$, where $H_{t}(z)$ is a continuous function of $t \geq 0$ for any $z \in \mathbb{C} \backslash \mathbb{R}$;

(4) a vector field $A(z)=\lim _{t \backslash 0}\left(H_{t}(z)-z\right) / t$ on the upper half plane which has the form

$$
A(z)=-\gamma+\int_{\mathbb{R}} \frac{1+x z}{x-z} d \tau(x),
$$

where $\gamma \in \mathbb{R}$ and $\tau$ is a positive finite measure.

The integral representation in (4) is the Lévy-Khinchin formula in monotone probability. The correspondence of (3) and (4) is obtained through the following ordinary differential equation (ODE):

$$
\frac{d}{d t} H_{t}(z)=A\left(H_{t}(z)\right), \quad H_{0}(z)=z,
$$

for $z \in \mathbb{C} \backslash \mathbb{R}$. The fact that the solution does not explode in finite time is a consequence of [9]. We call $A(z)$ the associated vector field. When $\tau$ has all moments, then the coefficients of the Laurent expansion of $A$ coincide with cumulants [14.

In this paper, we analyze monotone convolution semigroups, especially supports and moments, comparing the results with the classical, free and Boolean cases. We hope that our results will be of use in clarifying similarity and dissimilarity between monotone independence and other kinds of independence. This work is also expected to have connections with an operator-theoretic approach [12] and a categorical approach [11].

The contents of sections are as follows. In Section 2, we give a condition for a probability measure to be supported on the positive real line, and show how moments change under monotone convolution. In Section 3 , we derive a differential equation for the minimum of the support of a monotone convolution semigroup. In Section 4, we study how a property of a monotone convolution semigroup changes with respect to the time parameter. A timeindependent property of a convolution semigroup is one that is determined at an instant. We show that the following properties are time-independent: symmetry around 0 ; the concentration of the support on the positive real line; the lower boundedness of the support; the finiteness of a moment of even order. All these properties are also time-independent in classical convolution semigroups. In Section 5, a monotone analogue of the Bercovici-Pata 
bijection is defined. Many time-independent properties of the previous section can be formulated in terms of that bijection. In Section 6, we study convolution semigroups in free probability and Boolean probability. A remarkable point is that the concentration of the support on the positive real line is a time-independent property in the monotone, Boolean and classical cases, but not in free probability.

\section{Behavior of supports and moments under monotone convolu-}

tion. We consider properties of probability measures which are conserved under monotone convolution. Let $\mu$ be a probability measure. Define the minimum and the maximum of the support:

$$
a(\mu):=\inf \{x \in \operatorname{supp} \mu\}, \quad b(\mu):=\sup \{x \in \operatorname{supp} \mu\} .
$$

Here $-\infty \leq a(\mu)<\infty$ and $-\infty<b(\mu) \leq \infty$. We say that $\mu$ has an isolated

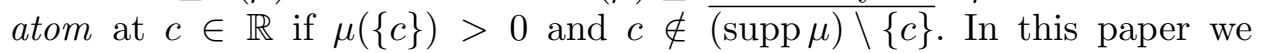
occasionally consider analytic continuations of functions such as $G_{\mu}$ or $H_{\mu}$ from $\mathbb{C} \backslash \mathbb{R}$ to an open subset $U$ of $\mathbb{C}$ which intersects $\mathbb{R}$. If no confusion can arise, for simplicity, we only say that a function is analytic in $U$, instead of saying that a function has an analytic continuation.

Lemma 2.1. Let $\mu$ be a probability measure. We use the notation (1.1).

(1) $(\operatorname{supp} \mu)^{c} \cup(\mathbb{C} \backslash \mathbb{R})$ is the maximal domain in which $G_{\mu}(z)$ is analytic. Similarly, $(\operatorname{supp} \eta)^{c} \cup(\mathbb{C} \backslash \mathbb{R})$ is the maximal domain in which $H_{\mu}(z)$ is analytic.

(2) $\left\{x \in(\operatorname{supp} \mu)^{c} ; G_{\mu}(x) \neq 0\right\} \subset(\operatorname{supp} \eta)^{c}$. Similarly, $\left\{x \in(\operatorname{supp} \eta)^{c}\right.$; $\left.H_{\mu}(x) \neq 0\right\} \subset(\operatorname{supp} \mu)^{c}$. In particular, $a(\eta) \geq a(\mu)$ since $G_{\mu}(x) \neq 0$ for $x \in(-\infty, a(\mu))$.

Proof. These statements easily follow from the Perron-Stieltjes inversion formula.

A classical infinitely divisible distribution necessarily has a non-compact support, except for a delta measure. The situation in the monotone, free and Boolean cases is different. For instance, a centered arcsine law is $\triangleright$ infinitely divisible. For this reason the study of the maximum or minimum of a support becomes important. It is known that if $\lambda=\nu \triangleright \mu$ and $\lambda$ has a compact support, then the support of $\mu$ is also compact [16]. We generalize this and prove a basic estimate on supports.

Proposition 2.2. For any probability measures $\nu$ and $\mu$ the following inequalities hold.

(1) If $\operatorname{supp} \nu \cap(-\infty, 0] \neq \emptyset$ and $\operatorname{supp} \nu \cap[0, \infty) \neq \emptyset$, then $a(\mu) \geq a(\nu \triangleright \mu)$ and $b(\mu) \leq b(\nu \triangleright \mu)$.

(2) If $\operatorname{supp} \nu \subset(-\infty, 0]$, then $a(\mu) \geq a(\nu \triangleright \mu)$ and $b(\nu)+b(\mu) \leq b(\nu \triangleright \mu)$. 
(3) If $\operatorname{supp} \nu \subset[0, \infty)$, then $a(\nu)+a(\mu) \geq a(\nu \triangleright \mu)$ and $b(\mu) \leq b(\nu \triangleright \mu)$.

Proof. For a probability measure $\rho$, we denote by $\rho^{x}$ the probability measure $\delta_{x} \triangleright \rho$. This is useful since $\nu \triangleright \mu$ can be expressed as

$$
\nu \triangleright \mu(B)=\int_{\mathbb{R}} \mu^{x}(B) \nu(d x)
$$

for Borel sets $B$ [16].

Let $\lambda:=\nu \triangleright \mu$. We first prove the following inequalities for an arbitrary probability measure $\rho$ :

$$
\begin{cases}a\left(\rho^{x}\right) \geq a(\rho), \quad b\left(\rho^{x}\right) \leq b(\rho)+x & \text { for all } x>0 \\ a\left(\rho^{x}\right) \geq a(\rho)-|x|, \quad b\left(\rho^{x}\right) \leq b(\rho) & \text { for all } x<0 .\end{cases}
$$

It is easy to prove that $\rho^{x}$ can be characterized by $G_{\rho^{x}}=G_{\rho} /\left(1-x G_{\rho}\right)$. If $x>0$, then $1-x G_{\rho}(z) \neq 0$ for $z \in \mathbb{C} \backslash[a(\rho), b(\rho)+x]$ and $G_{\rho}$ is analytic in this domain. Therefore, the first inequality holds. The second is proved similarly.

Let $J:=\operatorname{supp} \lambda$. In view of the relation $\lambda(A)=\int_{\mathbb{R}} \mu^{x}(A) d \nu(x)$, we have $\lambda\left(J^{c}\right)=\int_{\mathbb{R}} \mu^{x}\left(J^{c}\right) d \nu(x)=0$. Hence $\mu^{x}\left(J^{c}\right)=0, \nu$-a.e. $x \in \mathbb{R}$. Take any $x_{0}$ such that $\mu^{x_{0}}\left(J^{c}\right)=0$. Then $a\left(\mu^{x_{0}}\right) \geq a(\lambda)$ and $b\left(\mu^{x_{0}}\right) \leq b(\lambda)$. If $x_{0}>0$, combining the inequalities $a\left(\rho^{x}\right) \geq a(\rho)-|x|$ and $b\left(\rho^{x}\right) \leq b(\rho)$ for $\rho=\mu^{x_{0}}$ and $x=-x_{0}<0$, we obtain

$$
a(\mu)=a\left(\mu^{x_{0}-x_{0}}\right) \geq a(\lambda)-\left|x_{0}\right|, \quad b(\mu)=b\left(\mu^{x_{0}-x_{0}}\right) \leq b(\lambda) .
$$

Similarly if $x_{0}<0$,

$$
a(\mu) \geq a(\lambda), \quad b(\mu) \leq b(\lambda)+\left|x_{0}\right| .
$$

Assume that $\operatorname{supp} \nu \subset(-\infty, 0]$. Then $a(\mu) \geq a(\lambda)$ and $b(\mu) \leq b(\lambda)+|b(\nu)|$ since there is a sequence of such $x_{0}$ 's converging to $b(\nu)$. Hence we have proved (2). The statements (1) and (3) are proved in a similar way.

COROLlary 2.3. Let $\nu$ be a probability measure and let $n \geq 1$ be a natural number.

(1) If $\operatorname{supp}\left(\nu^{\triangleright n}\right) \subset(-\infty, 0]$, then $\operatorname{supp} \nu \subset(-\infty, 0]$ and $|b(\nu)| \geq$ $n^{-1}\left|b\left(\nu^{\triangleright n}\right)\right|$.

(2) If $\operatorname{supp}\left(\nu^{\triangleright n}\right) \subset[0, \infty)$, then $\operatorname{supp} \nu \subset[0, \infty)$ and $a(\nu) \geq n^{-1} a\left(\nu^{\triangleright n}\right)$.

This corollary puts a restriction on the support of a $\triangleright$-infinitely divisible distribution. The continuous time version of (2) will be proved in Section 4 .

Proof. Let $\lambda:=\nu^{\triangleright n}$.

(1) Assume that both $b(\nu)>0$ and $b(\lambda)=b\left(\nu^{\triangleright n}\right) \leq 0$. Then two cases are possible: (a) $\operatorname{supp} \nu \cap[0, \infty) \neq \emptyset$ and $\operatorname{supp} \nu \cap(-\infty, 0] \neq \emptyset$; (b) $\operatorname{supp} \nu \subset[0, \infty)$. We apply Proposition 2.2 replacing $\lambda$ and $\mu$ with $\nu^{\triangleright n}$ and $\nu^{\triangleright n-1}$, respectively. In both cases (a) and (b), we have $b\left(\nu^{\triangleright n-1}\right) \leq$ 
$b(\lambda) \leq 0$. This argument can be repeated and finally we have $b(\nu) \leq 0$, a contradiction. Therefore, $b(\nu) \leq 0$. By the iterative use of Proposition 2.2 (2) we obtain $b\left(\nu^{\triangleright n}\right) \geq n b(\nu)$, from which the conclusion follows. A similar argument applies to (2).

The following statement is well known. We will need almost the same argument in Proposition 2.5 .

Lemma 2.4. For a finite measure $\mu, \lim _{y \backslash 0} i y G_{\mu}(a+i y)=\mu(\{a\})$ for all $a \in \mathbb{R}$.

Proof. This follows from the dominated convergence theorem.

Now we prove a condition for a support to be included in the positive real line. A similar result was obtained in [6].

Proposition 2.5. We use the notation (1.1). Then $\operatorname{supp} \mu \subset[0, \infty)$ if and only if $\operatorname{supp} \eta \subset[0, \infty)$ and $H_{\mu}(-0) \leq 0$. Moreover, if $\operatorname{supp} \eta \subset[0, \infty)$, the condition $H_{\mu}(-0) \leq 0$ is equivalent to

$$
\eta(\{0\})=0, \quad \int_{0}^{\infty} \frac{1}{x} d \eta(x)<\infty, \quad b+\int_{0}^{\infty} \frac{1}{x} d \eta(x) \leq 0 .
$$

Proof. If supp $\eta \subset[0, \infty)$ and $H_{\mu}(-0) \leq 0$, we have $H_{\mu}(u)<0$ for all $u<0$ since $H_{\mu}$ is strictly increasing. Then $G_{\mu}=1 / H_{\mu}$ is analytic in $\mathbb{C} \backslash[0, \infty)$, which implies supp $\mu \subset[0, \infty)$. Conversely, assume $\operatorname{supp} \mu \subset[0, \infty)$. By Lemma 2.1. we have $\operatorname{supp} \eta \subset[0, \infty)$. If $H_{\mu}(-0)$ were greater than 0 , there would exist $u_{0}<0$ such that $H_{\mu}\left(u_{0}\right)=0$. Then $\mu$ has an atom at $u_{0}<0$, which contradicts the assumption. Therefore, $H_{\mu}(-0) \leq 0$.

We show the equivalence in the last claim. It is not difficult to prove that (*) implies $H_{\mu}(-0) \leq 0$. For the converse, assume that $\lambda:=\eta(\{0\})>0$. By a similar argument to Lemma 2.4, we can prove that $\lim _{u>0} u H_{\mu}(u)=-\lambda$. Therefore, for $u<0$ sufficiently close to 0 , we have $H_{\mu}(u)>-\lambda / 2 u>$ 0 , which contradicts the condition $H_{\mu}(-0) \leq 0$. Consequently, we have $\eta(\{0\})=0$. Since $f_{u}(x):=(1+x u) /(x-u)$ is increasing with respect to $u$, we can apply the monotone convergence theorem and obtain the two inequalities of $(*)$.

COROLlary 2.6. Monotone convolution preserves the set $\{\mu ; \operatorname{supp} \mu \subset$ $[0, \infty)\}$ of probability measures.

Proof. If $\operatorname{supp} \mu, \operatorname{supp} \nu \subset[0, \infty)$, then $H_{\mu \triangleright \nu}=H_{\mu} \circ H_{\nu}$ is analytic in $\mathbb{C} \backslash[0, \infty)$. Since $H_{\mu \triangleright \nu}$ is increasing in $(-\infty, 0)$, we have $H_{\mu \triangleright \nu}(-0)=$ $H_{\mu} \circ H_{\nu}(-0) \leq H_{\mu}(-0) \leq 0$. By Proposition 2.5, we obtain $\operatorname{supp}(\mu \triangleright \nu) \subset$ $[0, \infty)$.

REMARK 2.7. The above property is also true for Boolean convolution. The proof is similar. We note that the corollary follows immediately if we 
use the operator-theoretic realization of monotone independent random variables in [12].

Next we consider moments. Let $m_{n}(\mu):=\int_{\mathbb{R}} x^{n} \mu(d x)$ be the $n$th moment of a probablility measure $\mu$.

Proposition 2.8. Let $\mu$ be a probability measure and let $n \geq 1$ be a natural number. Then the following conditions are equivalent:

(1) $m_{2 n}(\mu)<\infty$;

(2) $H_{\mu}$ has the expression $H_{\mu}(z)=z+a+\int_{\mathbb{R}} \frac{\rho(d x)}{x-z}$, where $a \in \mathbb{R}$ and $\rho$ is a positive finite measure satisfying $m_{2 n-2}(\rho)<\infty$;

(3) there exist $a_{1}, \ldots, a_{2 n} \in \mathbb{R}$ such that

$$
\begin{aligned}
& \qquad H_{\mu}(z)=z+a_{1}+\frac{a_{2}}{z}+\cdots+\frac{a_{2 n}}{z^{2 n-1}}+o\left(|z|^{-(2 n-1)}\right) \\
& \text { for } z=i y(y \rightarrow \infty) .
\end{aligned}
$$

If (3) holds, for any $\delta>0$ the expansion (2.2) holds for $z \rightarrow \infty$ satisfying $\operatorname{Im} z>\delta|\operatorname{Re} z|$. Moreover, $a_{k+2}=-m_{k}(\rho)(0 \leq k \leq 2 n-2)$.

Proof. The equivalence $(1) \Leftrightarrow(3)$ follows from Theorem 3.2.1 in [1] by calculating the reciprocals. The implication $(2) \Rightarrow(3)$ is not difficult. The proof of $(3) \Rightarrow(2)$ uses the same technique as in Theorem 3.2.1 of [1].

Proposition 2.9. Let $\mu$ and $\nu$ be probability measures and let $n \geq 1$ be a natural number. If $m_{2 n}(\mu)<\infty$ and $m_{2 n}(\nu)<\infty$, then $m_{2 n}(\mu \triangleright \nu)<\infty$. Moreover,

$$
\begin{aligned}
& m_{l}(\mu \triangleright \nu) \\
& =m_{l}(\mu)+m_{l}(\nu)+\sum_{k=1}^{l-1} \sum_{\substack{j_{0}+j_{1}+\cdots+j_{k}=l-k \\
0 \leq j_{p}, 0 \leq p \leq k}} m_{k}(\mu) m_{j_{0}}(\nu) \cdots m_{j_{k}}(\nu)
\end{aligned}
$$

for $1 \leq l \leq 2 n$.

Proof. We note that $\operatorname{Im} H_{\nu}(z) \geq \operatorname{Im} z$. For any $\delta>0$, there exists $M=$ $M(\delta)>0$ such that

$$
\operatorname{Im} H_{\nu}(i y) \geq y>\delta\left|\operatorname{Re} H_{\nu}(i y)\right| \quad \text { for } y>M .
$$

By (2.2), we obtain

$$
\begin{aligned}
& H_{\mu}\left(H_{\nu}(i y)\right) \\
& \quad=H_{\nu}(i y)+a_{1}+a_{2} G_{\nu}(i y)+\cdots+a_{2 n} G_{\nu}(i y)^{2 n-1}+R\left(H_{\nu}(i y)\right),
\end{aligned}
$$

where $z^{2 n-1} R(z)=\int_{\mathbb{R}} \frac{x^{2 n-1}}{x-z} \rho(d x) \rightarrow 0$ as $z \rightarrow \infty$ with $\operatorname{Im} z>\delta|\operatorname{Re} z|$ for a fixed $\delta>0$. We have

$$
y^{2 n-1}\left|R\left(H_{\nu}(i y)\right)\right| \leq\left|H_{\nu}(i y)\right|^{2 n-1}\left|R\left(H_{\nu}(i y)\right)\right| \rightarrow 0
$$


as $y \rightarrow \infty$ by 2.4). Thus $R\left(H_{\nu}(i y)\right)=o\left(y^{-(2 n-1)}\right)$. Expanding $H_{\nu}(z)$ in the form 2.2 , we can see that there exist $c_{1}, \ldots, c_{2 n} \in \mathbb{R}$ such that

$H_{\mu}\left(H_{\nu}(z)\right)=z+c_{1}+\frac{c_{2}}{z}+\cdots+\frac{c_{2 n}}{z^{2 n-1}}+o\left(|z|^{-(2 n-1)}\right) \quad$ for $z=i y(y \rightarrow \infty)$.

Then the $2 n$th moment of $\mu \triangleright \nu$ is finite by Proposition 2.8. The equality (2.3) is obtained by the expansion of $G_{\mu \triangleright \nu}(z)=G_{\mu}\left(1 / G_{\nu}(z)\right)$.

3. Differential equations arising from monotone convolution semigroups. Let $\left\{\mu_{t}\right\}_{t>0}$ be a weakly continuous $\triangleright$-convolution semigroup with $\mu_{0}=\delta_{0}$. We denote $H_{\mu_{t}}$ by $H_{t}$ for simplicity. We sometimes write $H(t, z)$ to express explicitly that $H_{t}(z)$ is a function of two variables. By (1.1), $H_{t}$ can be expressed as

$$
H_{t}(z)=b_{t}+z+\int_{\mathbb{R}} \frac{1+x z}{x-z} d \eta_{t}(x),
$$

where, for each $t>0, a_{t}$ is a real number and $\eta_{t}$ is a finite positive measure. Throughout this paper we denote by $A(z)$ the associated vector field.

In this section, we prove the following properties of the minimum of the support of a convolution semigroup.

THEOREM 3.1. Let $\left\{\mu_{t}\right\}_{t \geq 0}$ be a weakly continuous $\triangleright$-convolution semigroup with $\mu_{0}=\delta_{0}$. Assume that for every $t>0, \mu_{t}$ is not a delta measure and $\mu_{t}=\lambda(t) \delta_{\theta(t)}+\nu_{t}$ with $\theta(t) \notin \operatorname{supp} \nu_{t}, \theta(t)=a\left(\mu_{t}\right)$ and $\lambda(t) \geq 0$.

(1) Assume $a(\tau)>0$. Then four cases are possible:

(A) If $A\left(u_{0}\right)=0$ for some $u_{0} \in[-\infty, 0)$ and $A(u)<0$ on $\left(-\infty, u_{0}\right)$ and $A(u)>0$ on $\left(u_{0}, 0\right)$ (when $u_{0}=-\infty$, we understand the condition as $A>0)$, then $\lambda(t)>0$. Moreover, $u_{0}<\theta(t)<0$ for all $t>0$.

(B) If $A(u)<0$ on $(-\infty, 0)$ and $A(0)=0$, then $\theta(t)=0$ and $\lambda(t)>0$ for all $t>0$.

(C) If there exists $u_{0} \in(0, a(\tau))$ such that $A(u)<0$ on $\left(-\infty, u_{0}\right)$ and $A(u)>0$ on $\left(u_{0}, a(\tau)\right)$, then $\theta(t) \in\left(0, u_{0}\right)$ and $\lambda(t)>0$ for $0<t$ $<\infty$.

(D) If $A(u)<0$ on $(-\infty, a(\tau))$, then there exists $t_{0} \in(0, \infty]$ such that $\lambda(t)>0$ for $0<t<t_{0}$ and $\lambda(t)=0$ for $t_{0} \leq t<\infty$.

If $A(0) \neq 0$ and $\lambda(t)>0$, then $\lambda(t)=A(\theta(t)) / A(0)$. If $A(0)=0$ (case (B)), then $\lambda(t)=e^{-A^{\prime}(0) t}$. For the position of the delta measure, the following ODE holds:

$$
\left\{\begin{array}{l}
\frac{d}{d t} \theta(t)=-A(\theta(t)), \\
\theta(0)=0 .
\end{array}\right.
$$


(2) Assume $a(\tau)>-\infty$. Then three cases can occur in terms of the signs of the associated vector field:

(a) $A(u)>0$ on $(-\infty, a(\tau))$;

(b) $A\left(u_{0}\right)=0$ for some $u_{0} \in(-\infty, a(\tau))$ and $A(u)<0$ on $\left(-\infty, u_{0}\right)$ and $A(u)>0$ on $\left(u_{0}, a(\tau)\right)$

(c) $A(u)<0$ on $(-\infty, a(\tau))$.

In cases (a) and (b), we have the following ODE for $a\left(\nu_{t}\right)$ :

$$
\left\{\begin{array}{l}
\frac{d}{d t} a\left(\nu_{t}\right)=-A\left(a\left(\nu_{t}\right)\right), \\
a\left(\nu_{0}\right)=a(\tau) .
\end{array}\right.
$$

In case (c), the equality $a\left(\nu_{t}\right)=a(\tau)$ holds for a.e. $t$ and $a\left(\nu_{t}\right) \geq a(\tau)$ for all $t \in[0, \infty)$. Moreover, if $\lim _{u \nearrow a(\tau)} A(u)<0$, then $a\left(\nu_{t}\right)=a(\tau)$ for all $t$.

EXAMPLE 3.2. We can confirm the validity of the ODEs for $\theta(t)$ and $a\left(\nu_{t}\right)$, and the validity of the formula for the weight for a delta measure, in each of the following examples.

- Arcsine law:

$$
\mu_{t}=\frac{1}{\pi \sqrt{2 t-x^{2}}} 1_{(-\sqrt{2 t}, \sqrt{2 t})}(x) d x, \quad A(z)=-\frac{1}{z},
$$

$a(\tau)=0, a\left(\mu_{t}\right)=-\sqrt{2 t}$.

- A deformation of $\alpha$-strictly stable distributions $(0<\alpha<2)$ with parameter $c \in \mathbb{C}, \operatorname{Im} c=0, \operatorname{Re} c \geq 0$ (see [13]):

$$
\mu_{t}=\mu_{t, \mathrm{ac}}, \quad \operatorname{supp} \mu_{t, \mathrm{ac}}=\left(-\infty, c+t^{1 / \alpha}\right], \quad A(z)=-\frac{1}{\alpha}(z-c)^{1-\alpha} .
$$

We can check that the solution of the ODE 3.3$)$ is $c+t^{1 / \alpha}$ (the same ODE (3.3) holds for $\left.b\left(\mu_{t}\right)\right)$.

- The monotone Poisson distribution with parameter $\lambda>0$ :

$$
\mu_{t}(d x)=\mu_{t, \text { ac }}+\mu_{t, \text { sing }}, \quad A(z)=\frac{\lambda z}{1-z},
$$

where $\mu_{t, \text { sing }}$ is a delta measure at 0 , and hence $A(0)=0$ and $A^{\prime}(0)=\lambda$. This is case (B). Therefore, $\mu_{t, \text { sing }}=e^{-\lambda t} \delta_{0}$.

3.1. Differential equation of the delta measure. We summarize three equalities, some of which were used by Muraki in [16].

LEMmA 3.3. Let $\left\{\mu_{t}\right\}_{t \geq 0}$ be a weakly continuous $\triangleright$-convolution semigroup with $\mu_{0}=\delta_{0}$. Then we have three equalities on $\mathbb{C} \backslash \mathbb{R}$ :

(1) $A\left(H_{t}(z)\right)=A(z) \frac{\partial H_{t}}{\partial z}(z)$;

(2) $\frac{\partial}{\partial t} G_{t}(z)=A(z) \frac{\partial}{\partial z} G_{t}(z)$;

(3) $\frac{\partial}{\partial t} H_{t}(z)=A(z) \frac{\partial}{\partial z} H_{t}(z)$. 
Proof. Since $H_{t}(z)$ is a flow in $\mathbb{C} \backslash \mathbb{R}, H_{t} \circ H_{s}=H_{t+s}$ for $t, s \geq 0$. Now, (1) follows by taking the derivative $\left.\frac{\partial}{\partial s}\right|_{s=0} ;$ (3) follows from (1) and 1.3 ; and (2) follows from (3) immediately.

First we treat a distribution which contains a delta measure at the minimum of the support. Suppose that $\left\{\mu_{t}\right\}_{t>0}$ is a weakly continuous $\triangleright$ convolution semigroup with $\mu_{0}=\delta_{0}$. Then $\mu$ can be written as $\mu=\lambda \delta_{\theta}+\nu$ with $\theta \in(\operatorname{supp} \nu)^{c}$ and $0<\lambda<1$. We use the integral representation in Theorem 1.1 (4) for the associated vector field $A(z)$. Throughout this subsection, we assume that $\tau \neq 0$ and $a(\tau)>0$. We shall show that there exists a delta measure at the minimum point of the support for some (finite or infinite) time interval. Moreover, the weight of the delta measure is calculated.

The derivative of $A$ satisfies $A^{\prime}(u)>0$ for all $u \in(-\infty, 0)$. This implies that five cases are possible:

(A) $A(u)>0$ on $(-\infty, 0)$;

$\left(\mathrm{A}^{\prime}\right) A\left(u_{0}\right)=0$ for some $u_{0} \in(-\infty, 0)$ and $A(u)<0$ on $\left(-\infty, u_{0}\right)$ and $A(u)>0$ on $\left(u_{0}, 0\right)$

(B) $A(u)<0$ on $(-\infty, 0)$ and $A(0)=0$;

(C) there exists $u_{0} \in(0, a(\tau))$ such that $A(u)<0$ on $\left(-\infty, u_{0}\right)$ and $A(u)>0$ on $\left(u_{0}, a(\tau)\right)$;

(D) $A(u)<0$ on $(-\infty, a(\tau))$.

We consider the solution of the ODE (1.3) on the real line as well as on $\mathbb{C} \backslash \mathbb{R}$.

Cases (A) And $\left(\mathrm{A}^{\prime}\right)$. Case (A) reduces to $\left(\mathrm{A}^{\prime}\right)$ if we define $u_{0}:=-\infty$. Since $H(t, u)$ is an increasing function of $u \in\left(\operatorname{supp} \eta_{t}\right)^{c}$, there is a unique point $\theta(t)$ satisfying $u_{0}<\theta(t)<0$ and

$$
H(t, \theta(t))=0 .
$$

$\theta(t)$ is a zero point of $H_{t}$ of degree 1 since $\partial_{u} H(t, u) \geq 1$. Therefore, by Lemma 2.4 there is a delta measure $\lambda(t) \delta_{\theta(t)}$ in $\mu_{t}$ with $u_{0}<\theta(t)<0$. By the implicit function theorem, $\theta(t)$ is of class $C^{\omega}$. Differentiating the equation $H(t, \theta(t))=0$ and using Lemma 3.3 , we obtain

$$
\theta^{\prime}(t)=-\frac{\frac{\partial H}{\partial t}(t, \theta(t))}{\frac{\partial H}{\partial z}(t, \theta(t))}=-A(\theta(t)) .
$$

The initial condition is $\theta(0)=0$.

Case (B). In this case, the same differential equation (3.5) holds. Since $A(0)=0$, we have $\theta(t)=0$ for all $t$. This is true for a monotone Poisson distribution. 
Cases (C) And (D). These cases can be treated at the same time. We define

$$
u_{1}:= \begin{cases}u_{0} & \text { in case (C), } \\ a(\tau) & \text { in case (D). }\end{cases}
$$

In both cases, $H_{t}$ is analytic in $\mathbb{C} \backslash\left[u_{1}, \infty\right)$ (see Subsection 3.2 for details). Then there exists $t_{0} \in(0, \infty]$ such that $\mu_{t}$ includes a delta measure in $\left(0, u_{1}\right)$ for $0<t<t_{0}$. We can prove that $t_{0}=\infty$ in case (C). In case (D), there is an example where $t_{0}<\infty$ holds (see the "Examples" section in [13]). Moreover, $t_{0}=\infty$ may occur if $\lim _{u / a(\tau)} A(u)=0$. The measure $\mu_{t}$ has the form

$$
\mu_{t}= \begin{cases}\lambda(t) \delta_{\theta(t)}+\nu_{t}, & 0 \leq t<t_{0}, \\ \nu_{t}, & t_{0} \leq t<\infty,\end{cases}
$$

where $0<\lambda(t) \leq 1$ and $0 \leq \theta(t)<a(\tau)$ for $0 \leq t<t_{0}$, and $a\left(\nu_{t}\right) \geq a(\tau)$ for all $0<t<\infty$. The differential equation (3.5) holds also in this case.

Weight $\lambda(t)$ in cases $(A),\left(A^{\prime}\right),(C)$ and $(D)$. It is possible to calculate the weight $\lambda(t)$. For the moment we skip case (B). Then we have $A(0) \neq 0$. We expand $H_{t}(z)$ in a Taylor series around $\theta(t)$ as $H_{t}(z)=\sum_{n=1}^{\infty} a_{n}(t)(z-$ $\theta(t))^{n}$ with $a_{1}(t)=1 / \lambda(t)$. Also we expand $A(z)$ as $\sum_{n=0}^{\infty} b_{n} z^{n}$ with $b_{n} \in \mathbb{R}$. If we compare the coefficients of the constant term in the ODE (1.3), we obtain $-\theta^{\prime}(t) a_{1}(t)=b_{0}=A(0)$. Hence

$$
\lambda(t)=\frac{A(\theta(t))}{A(0)} .
$$

Weight $\lambda(t)$ in case $(B)$. In case (B), we express the Taylor expansions of $H_{t}$ and $A(z)$ at 0 respectively by $H_{t}(z)=\sum_{n=1}^{\infty} a_{n}(t) z^{n}$ and $A(z)=$ $\sum_{n=1}^{\infty} b_{n} z^{n}$ with $a_{1}(t)=1 / \lambda(t)$ and $b_{1}=A^{\prime}(0)>0$. Comparing the coefficients of $z^{n}$ in the ODE (1.3), we obtain the equation $a_{1}^{\prime}(t)=A^{\prime}(0) a_{1}(t)$. Therefore, $a_{1}(t)=e^{A^{\prime}(0) t}$ by the initial condition $a_{1}(0)=1$. Thus we obtain

$$
\lambda(t)=e^{-A^{\prime}(0) t} .
$$

3.2. Differential equation of the non-atomic part. In the previous subsection we considered the case $a(\tau)>0$. Now we consider a more general case. We investigate $a\left(\mu_{t}\right)$ including the case where there is no isolated delta measure at $a\left(\mu_{t}\right)$. Assume that the lower bound $a(\tau)$ of the Lévy measure $\tau$ is finite: $-\infty<a(\tau)$. Three cases can occur:

(a) $A(u)>0$ on $(-\infty, a(\tau))$;

(b) $A\left(u_{0}\right)=0$ for some $u_{0} \in(-\infty, a(\tau))$ and $A(u)<0$ on $\left(-\infty, u_{0}\right)$ and $A(u)>0$ on $\left(u_{0}, a(\tau)\right)$;

(c) $A(u)<0$ on $(-\infty, a(\tau))$.

$\mu_{t}$ may contain an isolated delta measure at $a\left(\mu_{t}\right)$. If so, we write $\mu_{t}=$ $\lambda(t) \delta_{\theta(t)}+\nu_{t}$. We understand that $\lambda(t)=0$ if $\mu_{t}$ does not contain an atom at 
$a\left(\mu_{t}\right)$, or if it does but the atom is not isolated. The motion of the position $\theta(t)$ of a delta measure was clarified in the previous subsection. To investigate $a\left(\nu_{t}\right)$, we define $E:[0, \infty) \rightarrow(-\infty, a(\tau)]$ by

$$
E(t):= \begin{cases}\sup \left\{u \leq a(\tau) ; H_{t}(u)=a(\tau)\right\} & \text { in cases (a) and (b), } \\ a(\tau) & \text { in case (c) }\end{cases}
$$

for $t \in[0, \infty)$. The definition in cases (a) and (b) may seem unclear since $H_{t}(z)$ was only defined in $\mathbb{C} \backslash \mathbb{R}$. The precise definition is as follows. Since cases (a) and (b) can be treated in the same way, we explain only case (b). If $u \in\left(u_{0}, a(\tau)\right)$, let $R(u)$ be defined so that $H_{t}(u)$ exists for all $t \in(0, R(u))$ and $\lim _{t / R(u)} H_{t}(u)=a(\tau)$. We observe that $0<R(u)<\infty$ for $u \in$ $\left(u_{0}, a(\tau)\right)$. Moreover, $R$ is a bijection from $\left(u_{0}, a(\tau)\right)$ to $(0, \infty)$. Therefore, we can define a bijection $E(t):=R^{-1}(t)$, which we have denoted simply as $\sup \left\{u \leq a(\tau) ; H_{t}(u)=a(\tau)\right\}$.

$a\left(\nu_{t}\right)$ is characterized by the following result.

Lemma 3.4. Let $\mu$ be $a \triangleright$-infinitely divisible distribution. Then $\mu$ can be expressed in the form $\mu=\lambda \delta_{\theta}+\nu$, where $\theta=a(\mu)$ is an isolated atom. We understand that $\mu=\nu$ or $\lambda=0$ if $\mu$ does not contain an atom at $a(\mu)$, or if it does but the atom is not isolated. Then

$a(\nu)=a(\eta)=\sup \left\{x \in \mathbb{R} ; H_{\mu}\right.$ has an analytic continuation to $\left.\mathbb{C} \backslash[x, \infty)\right\}$

under the notation (1.1).

Proof. The latter equality follows from Lemma 2.1(1) immediately and we only need to prove that $a(\nu)=a(\eta)$. First, if $\lambda=0$ we can easily prove $a(\mu)=a(\eta)$ by Lemma 2.1 (2). Second, we assume that $\lambda>0$. We show that $a(\nu) \neq a(\eta)$ causes a contradiction. We notice first that the difference $a(\nu) \neq a(\eta)$ comes from the zero points of $H_{\mu}(x)$ or $G_{\mu}(x)$ by Lemma 2.1(2). If $a(\nu)<a(\eta)$, then $H_{\nu}(a(\nu))=0$. This, however, implies that $G_{\mu}$ contains two atoms at $a(\nu)$ and $\theta$, which contradicts infinite divisibility (see Theorem 3.5 in [13]). If $a(\nu)>a(\eta)$, then $G_{\nu}(a(\eta))=0$. Since $\frac{d}{d x} H_{\mu}(x) \geq 1$ in $(\operatorname{supp} \mu)^{c} \subset \mathbb{R}, H_{\mu}(x)$ is increasing. Therefore $\lim _{x / a(\eta)} H_{\mu}(x)=\infty$ and $\lim _{x \backslash a(\eta)} H_{\mu}(x)=-\infty$. Also, $\lim _{x \rightarrow-\infty} H_{\mu}(x)=-\infty$. These imply that there exist $x_{1}<a(\eta)$ and $x_{2}>a(\eta)$ such that $H_{\mu}\left(x_{1}\right)=H_{\mu}\left(x_{2}\right)$. By Rouché's theorem, there exist distinct $z_{1}, z_{2} \in \mathbb{C}$ with positive imaginary parts such that $H_{\mu}\left(z_{1}\right)=H_{\mu}\left(z_{2}\right)$ (this argument is similar to the proof of Theorem 3.5 in [13]); this contradicts the infinite divisibility again since the solution of (1.3) defines a flow of injective mappings.

REMARK 3.5. If $\mu$ is not $\triangleright$-infinitely divisible, the above property does not hold. For instance, if $\mu=\frac{1}{2}\left(\delta_{-1}+\delta_{1}\right)$, then $a(\nu)=1$ but $a(\eta)=0$.

We define $a\left(\nu_{0}\right):=a(\tau)$ so that $a\left(\nu_{t}\right)$ becomes a continuous function at 0 . 
THEOREM 3.6. In cases (a) and (b), the equality $E(t)=a\left(\nu_{t}\right)$ holds for all $t \in[0, \infty)$. In case (c), the equality holds under the further assumption $\lim _{u / a(\tau)} A(u)<0$.

Proof. We can prove this equality by considering the region in which $H_{t}(z)$ is analytic. We first consider cases (a) and (b). We prove that

$$
E(t)=\sup \left\{x \in \mathbb{R} ; H_{t} \text { has an analytic continuation to } \mathbb{C} \backslash[x, \infty)\right\} .
$$

By reductio ad absurdum we show that $H_{t}$ never has an analytic continuation beyond $E(t)$. If $H_{t}(z)$ had an analytic continuation to $\mathbb{C} \backslash[E(t)+\delta, \infty)$ for some $t>0$ and $\delta>0$, the following three facts would follow: the image of $H_{t}(u)$ includes $a(\tau)$ since $\partial H / \partial u \geq 1$ and $H(t, E(t))=a(\tau) ; H_{t}$ is injective in $\mathbb{C} \backslash[E(t)+\delta, \infty)$; we can take $\delta>0$ small enough so that $A(z)$ is analytic in $\mathbb{C} \backslash[E(t)+\delta, \infty)$ since $E(t)<a(\tau)$. Then by the equality $A\left(H_{t}(z)\right)=A(z) \frac{\partial H_{t}}{\partial z}(z)$ in $\mathbb{C} \backslash \mathbb{R}$, we conclude that $A(z)$ has an analytic continuation to the image of $H_{t}$. In particular, $A$ is analytic around the point $a(\tau)$, a contradiction. Therefore, $H_{t}$ cannot have an analytic continuation beyond $E(t)$.

Conversely, for any $u<E(t), H_{t}(z)$ has an analytic continuation to the region $\mathbb{C} \backslash[u+\delta, \infty)$ for some $\delta>0$ by the solution of the ODE (1.3). Thus the equality (3.6) holds.

The proof of the equality $E(t)=a\left(\nu_{t}\right)$ in case (c) under the assumption $\lim _{u \succ a(\tau)} A(u)<0$ is similar. For all $t>0$, we have $\lim _{u \succ a(\tau)} H_{t}(u)<a(\tau)$. Assume that $H_{t}(z)$ has an analytic continuation to $\mathbb{C} \backslash[E(t)+\delta, \infty)$ for some $t>0$ and $\delta>0$. We can take $\delta$ small enough such that $H_{t}(u) \in$ $(-\infty, a(\tau))$ for all $u \in(-\infty, a(\tau)+\delta)$. This contradicts the equality $A\left(H_{t}(z)\right)$ $=A(z) \frac{\partial H_{t}}{\partial z}(z)$.

In case (c), if $\lim _{u \nearrow a(\tau)} A(u)=0$, the question whether $E(t)=a\left(\nu_{t}\right)$ holds for all $t>0$ or not has not been clarified yet. A partial answer is given in the following proposition.

Proposition 3.7. In case (c) we have $a\left(\nu_{t}\right)=a(\tau)$ a.e. with respect to the Lebesgue measure on $[0, \infty)$ and $a\left(\nu_{t}\right) \geq a(\tau)$ for all $t>0$.

Proof. Step 1. First, we prove that if $\limsup _{t \rightarrow t_{0}} a\left(\nu_{t}\right) \geq a\left(\nu_{t_{0}}\right)$, then $A(z)$ is analytic in the region $\left(-\infty, a\left(\nu_{t_{0}}\right)\right)$ and $a\left(\nu_{t_{0}}\right)=a(\tau)\left(=E\left(t_{0}\right)\right)$. Fix $\epsilon \in(0,1)$. Take a sequence $\left\{t_{n}\right\}_{n=1}^{\infty}$ such that $a\left(\nu_{t_{n}}\right) \geq a\left(\nu_{t_{0}}\right)-\epsilon / 2$ for all $n \geq 1$ and define a sequence of analytic functions in $\left(-\infty, a\left(\nu_{t_{0}}\right)-\epsilon\right)$ by

$$
A_{n}^{\epsilon}(z):=\frac{H_{t_{n}}(z)-H_{t_{0}}(z)}{t_{n}-t_{0}}
$$

for $n \geq 1$. For any compact set $K \subset \mathbb{C} \backslash[a(\tau)-\epsilon, \infty)$, we can prove that the 
sequence $\left\{A_{n}^{\epsilon}\right\}$ is uniformly bounded on $K$ for sufficiently large $n$. Hence we obtain the analyticity of $\partial_{t} H\left(t_{0}, z\right)$ in $\left(-\infty, a\left(\nu_{t_{0}}\right)-\epsilon\right)$. Since $1>\epsilon>0$ is arbitrary, we conclude that $\partial_{t} H\left(t_{0}, z\right)$ is analytic in $\left(-\infty, a\left(\nu_{t_{0}}\right)\right)$. The function $A(z)$ has an analytic continuation from $\mathbb{C} \backslash \mathbb{R}$ to $\mathbb{C} \backslash\left[a\left(\nu_{t_{0}}\right), \infty\right)$ by the equality $A(z)=\partial_{t} H\left(t_{0}, z\right) / \partial_{z} H\left(t_{0}, z\right)$.

Now we show $a(\tau)=a\left(\nu_{t_{0}}\right)$. As explained before, the solution $H_{t}(z)$ of the ODE exists for all time and for any initial position $z \in \mathbb{C} \backslash[a(\tau), \infty)$. Therefore, $a\left(\nu_{t}\right) \geq a(\tau)$ for all $t \in[0, \infty)$. Moreover, $a(\tau) \geq a\left(\nu_{t_{0}}\right)$ by the analyticity of $A(z)$ in $\left(-\infty, a\left(\nu_{t_{0}}\right)\right)$.

Step 2. We note that $a\left(\nu_{t}\right)$ is Borel measurable. This is easy since the coefficients of the Taylor expansion of $H_{t}$ are measurable (by the Cauchy integral formula), and $a\left(\nu_{t}\right)$ can be expressed as their upper limit. We define a Borel set $B$ by

$B:=\{t \in[0, \infty)$; there exist $\epsilon=\epsilon(t)>0$ and $\eta=\eta(t)>0$ such that

$$
\left.\left|a\left(\nu_{t}\right)-a\left(\nu_{s}\right)\right|>\epsilon \text { for all } s \text { satisfying } 0<|s-t|<\eta\right\} .
$$

If $t \in B^{c}$, then $a\left(\nu_{t}\right)=E(t)$ by Step 1 . It is known that a Borel measurable function on an interval is continuous except on an open set with arbitrarily small Lebesgue measure, by Lusin's theorem (see [10]). Therefore, the Lebesgue measure of $B$ is 0 . The inequality $a\left(\nu_{t}\right) \geq a(\tau)$ was already mentioned in the proof of Step 1.

So far we have proved that $E(t)=a\left(\nu_{t}\right)$ in generic cases. Next we find an ODE for the function $E(t)$. For $\epsilon>0$ define an approximate family by

$$
E_{\epsilon}(t):=\sup \left\{u \leq a(\tau) ; H_{t}(u)=a(\tau)-\epsilon\right\} .
$$

This approximation is needed to use the implicit function theorem in the proof of Theorem 3.9 .

Lemma 3.8. In cases (a) and (b), $E_{\epsilon}$ and $E$ enjoy the following properties:

(1) $E_{\epsilon}<E$ for all $\epsilon \in(0,1)$. In addition, $E_{\epsilon}$ converges to $E$ pointwise as $\epsilon \rightarrow 0$.

(2) $\sup _{\epsilon>0, t \in I}\left|E_{\epsilon}(t)\right|<\infty$ for any compact set $I \subset[0, \infty)$.

The proof is easy and we omit it.

TheOrem 3.9. In cases (a) and (b), E(t) satisfies the ODE

$$
\left\{\begin{array}{l}
\frac{d}{d t} E(t)=-A(E(t)) \text { for } 0<t<\infty, \\
E(0)=a(\tau) .
\end{array}\right.
$$

In particular, $E$ is in $C^{\omega}(0, \infty) \cap C[0, \infty)$. 
Proof. We note that $\partial H / \partial u \geq 1$. Thus the implicit function theorem is applicable to the equation $H=a(\tau)-\epsilon$ because $H$ is defined in the open set $\{(t, u) ; 0<t<\infty,-\infty<u<E(t)\}$ which contains $\left(t, E_{\epsilon}(t)\right)$ for all $t$. Therefore, $E_{\epsilon}$ is in $C^{\omega}(0, \infty)$ and its derivative is

$$
\frac{d E_{\epsilon}}{d t}(t)=-\frac{\partial_{t} H\left(t, E_{\epsilon}(t)\right)}{\partial_{u} H\left(t, E_{\epsilon}(t)\right)}=-A\left(E_{\epsilon}(t)\right)
$$

by Lemma 3.3. After integrating the above, we take the limit $\epsilon \rightarrow 0$ using Lemma 3.8 , to obtain

$$
E(t)=\int_{t}^{t_{1}} A(E(s)) d s+E\left(t_{1}\right) .
$$

This implies that $E$ is in $C^{\omega}(0, \infty)$ and the ODE holds. The right continuity of $E$ at 0 follows from the fact that $\lim _{t \backslash 0} H_{t}(z)=z$.

\section{Time-dependent and time-independent properties of a mono-} tone convolution semigroup. In classical probability theory, it is often true that a property of a convolution semigroup $\mu_{t}$ is completely determined at an instant. Such a property is called time-independent. In this section, we exhibit such properties for monotone convolution semigroups.

LEMMA 4.1. Let $\left\{\mu_{t}\right\}_{t \geq 0}$ be a weakly continuous $\triangleright$-convolution semigroup with $\mu_{0}=\delta_{0}$, and $A(z)$ be the associated vector field. If there exists $t_{0}>0$ such that $\operatorname{supp} \mu_{t_{0}} \subset[0, \infty)$, then $A(z)$ is analytic in $\mathbb{C} \backslash[0, \infty)$.

Proof. We have supp $\mu_{t_{0} / n} \subset[0, \infty)$ by Corollary 2.3(1). Let $A_{n}(z)$ be defined by $A_{n}(z):=\left(H_{t_{0} / n}(z)-z\right) /\left(t_{0} / n\right)$. Then $A_{n}$ is analytic in $\mathbb{C} \backslash[0, \infty)$ since $\operatorname{supp} \eta_{t_{0} / n} \subset[0, \infty)$ from Lemma 2.1. By definition $A(z)=$ $\lim _{n \rightarrow \infty} A_{n}(z)$ for $z \in \mathbb{C} \backslash \mathbb{R}$. By Montel's theorem, it suffices to show that the RHS is uniformly bounded on each compact subset $K$ of $\mathbb{C} \backslash[0, \infty)$. Since $H_{t}(i)=b_{t}+i\left(1+\eta_{t}(\mathbb{R})\right)$ is differentiable, there exist $M, M^{\prime}>0$ such that $\eta_{t}(\mathbb{R}) / t \leq M$ and $\left|b_{t} / t\right| \leq M^{\prime}$ for all $t \in\left[0, t_{0}\right]$. Then

$$
\left|A_{n}(z)\right| \leq\left|\frac{n}{t_{0}} b_{t_{0} / n}\right|+\left|\int_{0}^{\infty} \frac{1+x z}{x-z} \frac{n}{t_{0}} \eta_{t_{0} / n}(x)\right| \leq M^{\prime}+L^{\prime}
$$

for all $n$ and $z \in K$, where $L^{\prime}>0$ is a constant depending only on $K$.

Using Proposition 2.5 and Lemma 4.1, one can prove the monotone analogue of the subordinator theorem. For the classical version, the reader is referred to Theorem 24.11 of [17].

THEOREM 4.2. Let $\left\{\mu_{t}\right\}_{t \geq 0}$ be a weakly continuous $\triangleright$-convolution semigroup with $\mu_{0}=\delta_{0}$. Then the following statements are equivalent:

(1) there exists $t_{0}>0$ such that $\operatorname{supp} \mu_{t_{0}} \subset[0, \infty)$; 
(2) $\operatorname{supp} \mu_{t} \subset[0, \infty)$ for all $0 \leq t<\infty$;

(3) $\operatorname{supp} \tau \subset[0, \infty), \tau(\{0\})=0, \int_{0}^{\infty} x^{-1} d \tau(x)<\infty$ and $\gamma \geq \int_{0}^{\infty} x^{-1} d \tau(x)$.

REMARK 4.3. (i) The equality $\tau(\{0\})=0$ in condition (3) means that there is no component of a Brownian motion in the Lévy-Khinchin formula.

(ii) The equivalence also holds in the classical and Boolean Lévy-Khinchin formulae. In the free case, however, (1) and (2) are not equivalent (see Section 6).

Proof of Theorem 4.2. We note that (3) is equivalent to $\left(3^{\prime}\right): A$ is analytic in $\mathbb{C} \backslash[0, \infty)$ and $A<0$ on $(-\infty, 0)$, by an argument in Proposition 2.5 .

$(1) \Rightarrow(2),\left(3^{\prime}\right)$ : If $\left\{\mu_{t}\right\}$ is a delta measure, then the statement follows immediately. Assume that $\mu_{t}$ is not a delta measure for some $t>0$. This is equivalent to assuming that $\mu_{t}$ is not a delta measure for all $t>0$. Then $\tau$ is a non-zero positive finite measure. $A(z)$ is analytic in $\mathbb{C} \backslash[0, \infty)$ by Lemma 4.1, and hence supp $\tau \subset[0, \infty)$. Three cases are possible: (a) $A(u)$ $>0$ on $(-\infty, 0)$; (b) $A\left(u_{0}\right)=0$ for some $u_{0} \in(-\infty, 0)$ and $A(u)<0$ on $\left(-\infty, u_{0}\right)$ and $A(u)>0$ on $\left(u_{0}, 0\right)$; (c) $A(u)<0$ on $(-\infty, 0)$.

In cases (a) and (b), we have $a\left(\mu_{t}\right)<0$ for all $t>0$ by Theorem 3.1(2). In case (c), we have $a\left(\mu_{t}\right) \geq a(\tau) \geq 0$ again by Theorem 3.1 (2). Hence only case (c) does not contradict the assumption.

$\left(3^{\prime}\right) \Rightarrow(1)$ : This was actually proved at the end of the proof of $(1) \Rightarrow(2)$.

We can prove that the lower boundedness of the support is determined at one instant.

THEOREM 4.4. Let $\left\{\mu_{t}\right\}_{t \geq 0}$ be a weakly continuous $\triangleright$-convolution semigroup with $\mu_{0}=\delta_{0}$. Then the following statements are equivalent:

(1) there exists $t_{0}>0$ such that $\operatorname{supp} \mu_{t_{0}}$ is bounded below;

(2) $\operatorname{supp} \mu_{t}$ is bounded below for all $0 \leq t<\infty$;

(3) $\operatorname{supp} \tau$ is bounded below.

REMARK 4.5. The same kind of theorem also holds in the free and Boolean cases. The classical case is exceptional since condition (3) has to be replaced by $\operatorname{supp} \tau \subset[0, \infty), \tau(\{0\})=0$ and $\int_{-1}^{1}|x|^{-1} d \tau(x)<\infty$ [17]. Therefore, the boundedness below is not mapped bijectively by the monotone analogue of the Bercovici-Pata bijection defined in Section 5.

Proof of Theorem 4.4. (1) $\Rightarrow(3)$ : When $a\left(\mu_{t_{0}}\right) \geq 0$, the claim follows from Theorem 4.2. We consider the case $a\left(\mu_{t_{0}}\right)<0$. By Proposition 2.2 , we have $a\left(\mu_{t}\right) \geq a\left(\mu_{t_{0}}\right)>-\infty$ for all $t \leq t_{0}$. By the same argument as in Lemma 4.1, one can show that $A$ is analytic in $\left(-\infty, a\left(\mu_{t_{0}}\right)\right)$.

$(3) \Rightarrow(2)$ : The lower boundedness of the support of $\mu_{t}$ for all $t \geq 0$ comes from Theorem 3.1 . 
Next we consider the symmetry around the origin. We say that a measure $\mu$ on the real line is symmetric if $\mu(d x)=\mu(-d x)$. The proof depends on the assumption of compact support. We have not been able to prove the result for all probability measures.

THEOREM 4.6. Let $\left\{\mu_{t}\right\}_{t \geq 0}$ be a weakly continuous $\triangleright$-convolution semigroup with $\mu_{0}=\delta_{0}$. Assume that the support of each $\mu_{t}$ is compact (this is a time-independent property). Then the following statements are equivalent:

(1) there exists $t_{0}>0$ such that $\mu_{t_{0}}$ is symmetric;

(2) $\mu_{t}$ is symmetric for all $t>0$;

(3) $\gamma=0$ and $\tau$ is symmetric.

Proof. We prove this theorem in terms of moments. We use the representation of the vector field $A(z)=-\gamma+\int \frac{1}{x-z} d \sigma(x), d \sigma(x)=\left(1+x^{2}\right) d \tau(x)$, where $\sigma$ has a compact support. We write $m_{n}(t)=m_{n}\left(\mu_{t}\right)$ for simplicity. We notice that the symmetry is equivalent to the vanishing of odd moments for a compactly supported measure. Define a sequence $\left\{r_{n}\right\}_{n=1}^{\infty}$ by $r_{1}:=\gamma$, $r_{n}:=m_{n-2}(\sigma)$ for $n \geq 2$. Then $A(z)=-\sum_{n=1}^{\infty} r_{n} / z^{n-1}$. By Lemma 3.3 (2), we get the differential equations $d m_{0}(t) / d t=0$ and

$$
\frac{d m_{n}(t)}{d t}=\sum_{k=1}^{n} k r_{n-k+1} m_{k-1}(t) \quad \text { for } n \geq 1
$$

with initial conditions $m_{0}(0)=1$ and $m_{n}(0)=0$ for $n \geq 1$.

Now we prove the implications $(1) \Rightarrow(2)$ and $(1) \Rightarrow(3)$. We can easily show that $m_{2 n+1}\left(t_{0}\right)=0$ and $r_{2 n+1}=0$ for $n \geq 0$, and so $m_{2 n+1}(t)=0$ for all $t>0$ and $n \geq 0$. Then $\sigma$ and $\mu_{t}$ are both symmetric for all $t>0$. The proof of $(3) \Rightarrow(2)$ is similar.

We now exhibit some time-dependent properties.

Proposition 4.7.

(1) Absolute continuity is a time-dependent property.

(2) Existence of an atom is a time-dependent property.

Proof. Consider the following example [13]. Let $\left\{\mu_{t}\right\}_{t \geq 0}$ be the monotone convolution semigroup defined by

$$
H_{t}^{(\alpha, 1, c)}(z)=c+\left\{(z-c)^{\alpha}+t\right\}^{1 / \alpha} \quad \text { for } 0<\alpha<1 .
$$

Then $\mu_{t}$ contains an atom for $0 \leq t<|c|^{\alpha}$ and $\mu_{t}$ is absolutely continuous for $t \geq|c|^{\alpha}$.

The property $m_{2 n}(\mu)=\int_{\mathbb{R}} x^{2 n} \mu(d x)<\infty$ is also time-independent. That is, we prove the following theorem which is also true for the classical and 
free probabilities [5, 18. In addition, it also extends Theorem 4.9 in 16 to higher order moments.

THEOREM 4.8. Let $\left\{\mu_{t}\right\}_{t>0}$ be a weakly continuous $\triangleright$-convolution semigroup with $\mu_{0}=\delta_{0}$ and let $n \geq 1$ be a natural number. Then the following statements are equivalent:

(1) there exists $t_{0}>0$ such that $m_{2 n}\left(t_{0}\right)<\infty$;

(2) $m_{2 n}(t)<\infty$ for all $0<t<\infty$;

(3) $m_{2 n}(\tau)<\infty$.

Proof. (1) $\Rightarrow(2)$ : We use the notation $\mu_{t}^{y}:=\delta_{y} \triangleright \mu_{t}$ introduced in (2.1). For $0 \leq t \leq t_{0}$, we set $\lambda=\mu_{t_{0}-t}$ and $\nu=\mu_{t}$. Then $\iint x^{2 n} \mu_{t}^{y}(d x) \mu_{t_{0}-t}(d y)=$ $\int_{\mathbb{R}} x^{2 n} \mu_{t_{0}}(d x)<\infty$, which implies $m_{2 n}\left(\mu_{t}^{y}\right)<\infty$ for some $y \in \mathbb{R}$. By Proposition 2.8 , we obtain $m_{2 n}(t)<\infty$ for $0 \leq t \leq t_{0}$. For arbitrary $0<s<\infty$, we can write $s=k t_{0}+t$ with $k \in \mathbb{N}$ and $0 \leq t<t_{0}$. Then $m_{2 n}(s)<\infty$ by Proposition 2.9.

$(2) \Rightarrow(3)$ : We first note that $m_{k}(t)$ is a Borel measurable function of $t \geq 0$ since $\mu_{t}$ is weakly continuous. Moreover, we show that there exist $r_{1}, \ldots, r_{2 n} \in \mathbb{R}$ such that

$$
m_{l}(t)=\sum_{k=1}^{l} \sum_{1=i_{0}<i_{1}<\cdots<i_{k-1}<i_{k}=l+1} \frac{t^{k}}{k !} \prod_{p=1}^{k} i_{p-1} r_{i_{p}-i_{p-1}}
$$

for $1 \leq l \leq 2 n$. For the proof we use the equality

$$
\begin{aligned}
& m_{l}(t+s) \\
& \quad=m_{l}(t)+m_{l}(s)+\sum_{k=1}^{l-1} \sum_{\substack{j_{0}+j_{1}+\cdots+j_{k}=l-k \\
0 \leq j_{p}, 0 \leq p \leq k}} m_{k}(t) m_{j_{0}}(s) \cdots m_{j_{k}}(s)
\end{aligned}
$$

for $1 \leq l \leq 2 n$. For $l=1$, (4.4) becomes $m_{1}(t+s)=m_{1}(t)+m_{1}(s)$. This is Cauchy's functional equation and there exists $r_{1} \in \mathbb{R}$ such that $m_{1}(t)=r_{1} t$ by measurability (for a simple proof of this fact, see [2]). We assume that there exist $r_{1}, \ldots, r_{q} \in \mathbb{R}$ such that $(4.3)$ holds for $1 \leq l \leq q$. For an arbitrary $r_{q+1}^{\prime} \in \mathbb{R}$, we define

$$
\widetilde{m}_{q+1}(t):=r_{q+1}^{\prime} t+\sum_{k=2}^{q+1} \sum_{1=i_{0}<i_{1}<\cdots<i_{k-1}<i_{k}=q+2} \frac{t^{k}}{k !} \prod_{p=1}^{k} i_{p-1} r_{i_{p}-i_{p-1}} .
$$

Then

$$
\begin{aligned}
\widetilde{m}_{q+1}(t+s)= & \widetilde{m}_{q+1}(t)+\widetilde{m}_{q+1}(s) \\
& +\sum_{k=1}^{q} \sum_{\substack{j_{0}+j_{1}+\cdots+j_{k}=q+1-k \\
0 \leq j_{l}, 0 \leq l \leq k}} m_{k}(t) m_{j_{0}}(s) \cdots m_{j_{k}}(s) ;
\end{aligned}
$$


this will be proved in Proposition 4.10. Therefore, (4.4) and (4.6) imply that $m_{q+1}(t+s)-\widetilde{m}_{q+1}(t+s)=m_{q+1}(t)-\widetilde{m}_{q+1}(t)+m_{q+1}(s)-\widetilde{m}_{q+1}(s)$. This is again Cauchy's functional equation, and hence there exists $r_{q+1}^{\prime \prime} \in \mathbb{R}$ such that $m_{q+1}(t)=\widetilde{m}_{q+1}(t)+r_{q+1}^{\prime \prime} t$. The above argument runs until $q=2 n-1$, and thus we conclude that there exist $r_{1}, \ldots, r_{2 n} \in \mathbb{R}$ such that 4.3 holds for $1 \leq l \leq 2 n$.

From the equality $\frac{\partial G}{\partial t}(t, z)=A(z) \frac{\partial G}{\partial z}(t, z)$ we obtain

$$
A(z)=\frac{G(1, z)-\frac{1}{z}}{\int_{0}^{1} \frac{\partial G}{\partial z}(s, z) d s}
$$

which implies

$$
A(z)=-\frac{\frac{m_{1}(1)}{z^{2}}+\cdots+\frac{m_{2 n}(1)}{z^{2 n+1}}+o\left(|z|^{-(2 n+1)}\right)}{\frac{1}{z^{2}}+\frac{2 \int_{0}^{1} m_{1}(s) d s}{z^{3}}+\cdots+\frac{(2 n+1) \int_{0}^{1} m_{2 n}(s) d s}{z^{2 n+2}}+\int_{0}^{1} R_{s}(z) d s},
$$

where

$$
R_{s}(z)=\frac{2 n+1}{z^{2 n+2}} \int_{\mathbb{R}} \frac{x^{2 n+1}}{z-x} \mu_{s}(d x)+\frac{1}{z^{2 n+1}} \int_{\mathbb{R}} \frac{x^{2 n+1}}{(z-x)^{2}} \mu_{s}(d x) .
$$

Since $m_{2 n}(s)$ is a polynomial, $x^{2 n}$ is integrable with respect to the measure $\mu_{s}(d x) d s$ on $\mathbb{R} \times[0, t]$. We can easily show that $\int_{0}^{1} R_{s}(i y) d s=o\left(y^{-(2 n+2)}\right)$ by the dominated convergence theorem. Therefore, there exist $u_{1}, \ldots, u_{2 n} \in \mathbb{R}$ such that

$$
A(i y)=u_{1}+\frac{u_{2}}{i y}+\cdots+\frac{u_{2 n}}{(i y)^{2 n-1}}+o\left(y^{-(2 n-1)}\right) .
$$

By Proposition 2.8, we have $m_{2 n}(\tau)<\infty$ (the equivalence between (2) and (3) in Proposition 2.8 is true for $A(z)$ : the proof needs no changes).

$(3) \Rightarrow(2)$ : Since $m_{2 n}(\tau)<\infty$, we have the expansion

$$
A(z)=u_{1}+\frac{u_{2}}{z}+\cdots+\frac{u_{2 n}}{z^{2 n-1}}+Q(z),
$$

where

$$
Q(z):=\frac{1}{z^{2 n-1}} \int_{\mathbb{R}} \frac{x^{2 n-1}}{x-z}\left(1+x^{2}\right) \tau(d x) .
$$

We obtain

$$
H_{t}(z)=z+u_{1} t+\int_{0}^{t} \frac{u_{2}}{H_{s}(z)} d s+\cdots+\int_{0}^{t} \frac{u_{2 n}}{H_{s}(z)^{2 n-1}} d s+\int_{0}^{t} Q\left(H_{s}(z)\right) d s
$$

from the equality $\frac{d}{d t} H_{t}(z)=A\left(H_{t}(z)\right)$. We can prove that

$$
\sum_{k=p}^{2 n-1} \int_{0}^{t} \frac{u_{k+1}}{H_{s}(i y)^{k}} d s+\int_{0}^{t} Q\left(H_{s}(i y)\right) d s=o\left(y^{-(p-1)}\right)
$$


since $\left|\int_{0}^{t} H_{s}(i y)^{-k} d s\right| \leq t / y^{k}$. In addition, $\int_{0}^{t} Q\left(H_{s}(i y)\right) d s=o\left(y^{-(2 n-1)}\right)$ for any $t>0$ by the dominated convergence theorem.

Now we show by induction that there exist polynomials $c_{k}(t)$ of $t(1 \leq$ $k \leq 2 n)$ such that

$$
H_{t}(z)=z+c_{1}(t)+\frac{c_{2}(t)}{z}+\cdots+\frac{c_{2 n}(t)}{z^{2 n-1}}+o\left(|z|^{-(2 n-1)}\right) \quad(z=i y, y \rightarrow \infty)
$$

for any $t>0$. First $H_{t}(i y)=i y+u_{1} t+\frac{u_{2} t}{i y}+o\left(\frac{1}{y}\right)$ by 4.8$)$. Next we assume that there exist polynomials $c_{k}(t)(1 \leq k \leq 2 q)$ such that

$$
H_{t}(z)=z+c_{1}(t)+\frac{c_{2}(t)}{z}+\cdots+\frac{c_{2 q}(t)}{z^{2 q-1}}+P_{t}(z),
$$

where $P_{t}(i y)=o\left(y^{-(2 q-1)}\right)$ for any $t>0$. We can write

$$
P_{t}(z)=\frac{1}{z^{2 q-1}} \int_{\mathbb{R}} \frac{x^{2 q-1}}{x-z} \rho_{t}(d x),
$$

where $\rho_{t}$ is the positive finite measure of Proposition 2.8(2). Then we obtain the asymptotic behavior $\int_{0}^{t} P_{s}(i y) d s=o\left(y^{-(2 q-1)}\right)$. Substituting 4.10 into the right hand side of (4.8), we obtain the expansion

$$
H_{t}(z)=z+b_{1}(t)+\frac{b_{2}(t)}{z}+\cdots+\frac{b_{2 q+2}(t)}{z^{2 q+1}}+o\left(|z|^{-(2 q+1)}\right),
$$

where $b_{k}(t)$ is a polynomial of $t$ (we note that $b_{k}(t)=c_{k}(t)$ for $1 \leq k \leq 2 q$ by the uniqueness of the expansion). This induction goes until $q=n-1$ and we obtain (4.9). The conclusion follows from Proposition 2.8.

REMARK 4.9. In the proof of $(2) \Rightarrow(3)$ we have proved that $m_{k}(t)$ is a polynomial of $t$. This property might seem to be too strong: what we needed was the integrability of $m_{k}(t)$ in a finite interval. The author however could not find an alternative proof of the integrability.

The following result completes the above theorem.

Proposition 4.10. For any complex numbers $r_{n}, n \geq 1$, the functions $m_{n}(t)$ defined by

$$
m_{n}(t)=\sum_{k=1}^{n} \sum_{1=i_{0}<i_{1}<\cdots<i_{k-1}<i_{k}=n+1} \frac{t^{k}}{k !} \prod_{p=1}^{k} i_{p-1} r_{i_{p}-i_{p-1}}
$$

satisfy the equality

$$
\begin{aligned}
& m_{n}(t+s) \\
& =m_{n}(t)+m_{n}(s)+\sum_{k=1}^{n-1} \sum_{\substack{j_{0}+j_{1}+\cdots+j_{k}=n-k \\
0 \leq j_{p}, 0 \leq p \leq k}} m_{k}(t) m_{j_{0}}(s) \cdots m_{j_{k}}(s)
\end{aligned}
$$

for any $n \geq 1$. 
Proof. Every series in this proof is a formal power series. We define $A(z)=-\sum_{z=1}^{\infty} r_{n} / z^{n-1}$. We solve the differential equation 1.3 in the sense of formal power series. There exists a unique solution of the form $H_{t}(z)=\sum_{n=-1}^{\infty} a_{n}(t) / z^{n}$. It is easy to prove that $H_{t+s}(z)=H_{t}\left(H_{s}(z)\right)$ in the sense of formal power series with respect to $t, s, z$. If we define $G_{t}(z)$ to be $1 / H_{t}(z)$, then Lemma 3.3 holds with the same proof. We can easily prove that $m_{n}(t)$ are given by $G_{t}(z)=\sum_{n=0}^{\infty} m_{n}(t) / z^{n+1}$ using Lemma $3.3(2)$. Finally, 4.13 follows from the power series expansion of $G_{t+s}(z)$ $=G_{t}\left(1 / G_{s}(z)\right)$.

5. Connection to infinite divisibility in classical probability. Now we consider the correspondence between classical and monotone probability. The usual Lévy-Khinchin formula is given by

$$
\widehat{\mu}(u)=\exp \left(i \gamma u+\int_{\mathbb{R}}\left(e^{i x u}-1-\frac{i x u}{1+x^{2}}\right) \frac{1+x^{2}}{x^{2}} \tau(d x)\right),
$$

where $\gamma \in \mathbb{R}$ and $\tau$ is a positive finite measure. We show that the identification of $(\gamma, \tau)$ in Theorem 1.1 and in (5.1) is important. For instance, the support of a classical infinitely divisible distribution is concentrated on the positive real line if and only if (see Theorem 24.11 in [17])

$$
\operatorname{supp} \tau \subset[0, \infty), \quad \tau(\{0\})=0, \quad \int_{0}^{1} \frac{1}{x} \tau(d x)<\infty, \quad \gamma \geq \int_{0}^{\infty} \frac{1}{x} \tau(d x) .
$$

These conditions are exactly the same as in Theorem 4.2. Then it is natural to define the monotone analogue of the Bercovici-Pata bijection (for the details on the Bercovici-Pata bijection in free probability, the reader is referred to [7]). Let $\operatorname{ID}(\triangleright)$ be the set of all $\triangleright$-infinitely divisible distributions; let $\operatorname{ID}(*)$ be the set of all classical infinitely divisible distributions. We define a map $\Lambda_{M}: \operatorname{ID}(*) \rightarrow \operatorname{ID}(\triangleright)$ by sending the pair $(\gamma, \tau)$ in (5.1) to the pair $(\gamma, \tau)$ in Theorem 1.1 $(4)$. This map enjoys nice properties. Let $D_{\lambda}$ be the dilation operator defined by

$$
\int_{\mathbb{R}} f(x) D_{\lambda} \mu(d x)=\int_{\mathbb{R}} f(\lambda x) \mu(d x)
$$

for all probability measures $\mu$ and all bounded continuous functions $f$.

TheOREM 5.1. $\Lambda_{M}$ has the following properties:

(1) $\Lambda_{M}$ is continuous.

(2) $\Lambda_{M}\left(\delta_{a}\right)=\delta_{a}$ for all $a \in \mathbb{R}$.

(3) $D_{\lambda} \circ \Lambda_{M}=\Lambda_{M} \circ D_{\lambda}$ for all $\lambda>0$.

(4) $\Lambda_{M}$ maps the Gaussian with mean 0 and variance $\sigma^{2}$ to the arcsine law with mean 0 and variance $\sigma^{2}$. 
(5) $\Lambda_{M}$ maps the Poisson distribution with parameter $\lambda$ to the monotone Poisson distribution with parameter $\lambda$;

(6) $\Lambda_{M}$ gives a one-to-one correspondence between the set $\{\mu \in \operatorname{ID}(*)$; $\operatorname{supp} \mu \subset[0, \infty)\}$ and the set $\{\nu \in \operatorname{ID}(\triangleright) ; \operatorname{supp} \nu \subset[0, \infty)\}$.

(7) For all $\alpha \in(0,2), \Lambda_{M}$ gives a one-to-one correspondence between strictly $\alpha$-stable distributions and monotone strictly $\alpha$-stable distributions.

(8) If $\operatorname{supp} \tau$ is compact, the symmetry of $\mu \in \operatorname{ID}(*)$ is equivalent to the symmetry of $\Lambda_{M}(\mu)$.

(9) For each $n \geq 1, \Lambda_{M}$ gives a one-to-one correspondence between the set $\left\{\mu \in \operatorname{ID}(*) ; \int_{\mathbb{R}} x^{2 n} \mu(d x)<\infty\right\}$ and the set $\{\nu \in \operatorname{ID}(\triangleright)$; $\left.\int_{\mathbb{R}} x^{2 n} \nu(d x)<\infty\right\}$.

REMARK 5.2. Since monotone convolution is non-commutative, $\Lambda_{M}$ does not preserve the convolution structure: $\Lambda_{M}(\mu * \lambda) \neq \Lambda_{M}(\mu) \triangleright \Lambda_{M}(\lambda)$ for some $\mu, \lambda$.

Proof of Theorem 5.1. It is known that the convergence of a sequence $\left\{\mu_{n}\right\} \subset \mathrm{ID}(*)$ to some $\mu$ implies the convergence of the corresponding pair $\left(\gamma_{n}, \tau_{n}\right)$ to some $(\gamma, \tau)$. Now we have the family of ODEs driven by

$$
A_{n}(z)=-\gamma_{n}+\int_{\mathbb{R}} \frac{1+x z}{x-z} d \tau_{n}(x) ;
$$

we denote the flow by $\left\{H_{n, t}\right\}$. Since $\left(\gamma_{n}, \tau_{n}\right)$ converges to $(\gamma, \tau), A_{n}$ converges locally uniformly to $A$. By the basic result of the theory of ODE, it follows that $H_{n, 1}(z) \rightarrow H_{1}(z)$ locally uniformly, which implies that $\mu_{n}$ converges weakly to $\mu$.

(2), (4) and (5) are proved easily by using the Lévy-Khinchin formulae [16, 17].

(3) and (7) follow from direct computations of the Lévy-Khinchin formula. See [13] and [17].

Property (6) follows from Theorem 4.2 .

(8) and (9) are direct consequences of Theorems 4.6 and 4.8.

6. Time-independent properties of free and Boolean convolution semigroups. We prepare tools to study convolution semigroups in free and Boolean probabilities. The notation is chosen in order that the correspondence becomes clear among the Bercovici-Pata bijections in free, monotone and Boolean probability theories. We define

$$
K_{\mu}(z):=z-H_{\mu}(z)=\gamma-\int \frac{1+x z}{x-z} d \tau(x) .
$$


As proved in [19], the Boolean convolution $\mu \uplus \nu$ of probability distributions $\mu$ and $\nu$ is characterized by

$$
K_{\mu \uplus \nu}=K_{\mu}+K_{\nu} .
$$

Every probability measure is Boolean infinitely divisible.

We summarize results on infinitely divisible distributions in free probability (see [3, 8, for instance). For a probability measure $\mu$, there exist $\eta>0$ and $M>0$ such that $H_{\mu}$ has an analytic right inverse $H_{\mu}^{-1}$ defined on the region

$$
\Gamma_{\eta, M}:=\{z \in \mathbb{C} ;|\operatorname{Re} z|<\eta|\operatorname{Im} z|,|\operatorname{Im} z|>M\} .
$$

The Voiculescu transform $\phi_{\mu}$ is defined by $\phi_{\mu}(z):=H_{\mu}^{-1}(z)-z$ in the region where $H_{\mu}^{-1}$ is defined. For probability measures $\mu$ and $\nu$, the free convolution of $\mu$ and $\nu$ is characterized by the relation

$$
\phi_{\mu \boxplus \nu}=\phi_{\mu}+\phi_{\nu}
$$

TheOREM 6.1. Let $\mu$ be a probability measure on $\mathbb{R}$. Then $\mu$ is $\boxplus$-infinitely divisible if and only if there exist a finite measure $\tau$ and a real number $\gamma$ such that

$$
\phi_{\mu}(z)=\gamma+\int_{\mathbb{R}} \frac{1+x z}{z-x} d \tau(x) \quad \text { for } z \in \mathbb{C} \backslash \mathbb{R} .
$$

In this section we prove time-independent properties of free and Boolean convolution semigroups to clarify similarity and dissimilarity of the Bercovici-Pata bijections for free, Boolean and monotone convolutions. First we show that the subordinator theorem is valid in the Boolean case but not in the free case.

TheOREM 6.2. Let $\left\{\mu_{t}\right\}_{t \geq 0}$ be a weakly continuous Boolean convolution semigroup with $\mu_{0}=\delta_{0}$. Then the following statements are equivalent:

(1) there exists $t_{0}>0$ such that $\operatorname{supp} \mu_{t_{0}} \subset[0, \infty)$;

(2) $\operatorname{supp} \mu_{t} \subset[0, \infty)$ for all $0 \leq t<\infty$;

(3) $\operatorname{supp} \tau \subset[0, \infty), \tau(\{0\})=0, \int_{0}^{\infty} x^{-1} d \tau(x)<\infty$ and $\gamma \geq \int_{0}^{\infty} x^{-1} d \tau(x)$.

This type of theorem does not hold in free probability: condition (1) is not equivalent to (2).

Proof. In the Boolean case, the proof is easy by Proposition 2.5. In free probability, we give an example of a convolution semigroup for which (1) does not imply (2). Since the problem is symmetric around the origin, we show a counterexample concerning the condition $\operatorname{supp} \mu_{t} \subset(-\infty, 0]$. We define $\phi_{\mu}(z):=a-(z-c)^{1 / 2}$ with $a, c \in \mathbb{R}$. Then the corresponding convolution semigroup $\left\{\mu_{t}\right\}_{t \geq 0}$ with $\mu_{1}=\mu, \mu_{0}=\delta_{0}$ is characterized by

$$
H_{t}(z)=z-a t+t^{2} / 2+t \sqrt{z-\left(a t-t^{2} / 4+c\right)} .
$$


It is easy to show that $\operatorname{supp} \mu_{t} \subset(-\infty, 0]$ for sufficiently large $t$, but $\operatorname{supp} \mu_{t} \nsubseteq(-\infty, 0]$ for small $t$.

Symmetry around the origin is a time-independent property also in the cases of Boolean and free independence. The proof is easy.

Proposition 6.3. Let $\left\{\mu_{t}\right\}_{t \geq 0}$ be a weakly continuous Boolean (free) convolution semigroup with $\mu_{0}=\delta_{0}$. Then the following statements are equivalent.

(1) there exists $t_{0}>0$ such that $\mu_{t_{0}}$ is symmetric;

(2) $\mu_{t}$ is symmetric for all $t>0$;

(3) $\gamma=0$ and $\tau$ is symmetric.

We can also show that the property $\int_{\mathbb{R}} x^{2 n} d \mu_{t}(x)<\infty$ is time-independent in the Boolean case. In free probability, this result has recently been obtained in [5].

Proposition 6.4. Let $n \geq 1$ be a natural number. For a weakly continuous Boolean convolution semigroup $\left\{\mu_{t}\right\}_{t \geq 0}$, the following statements are equivalent:

(1) $\int_{\mathbb{R}} x^{2 n} d \mu_{t}(x)<\infty$ for some $t>0$;

(2) $\int_{\mathbb{R}} x^{2 n} d \mu_{t}(x)<\infty$ for all $t>0$;

(3) $\int_{\mathbb{R}} x^{2 n} d \tau(x)<\infty$.

Proof. This follows from Proposition 2.8.

Now we can compare the properties of the Bercovici-Pata bijections in free, monotone and Boolean probability theories. Boolean (strictly) stable distributions have been classified in [19], and they have the same characterization as in the monotone case. Considering the material of this section, we obtain the Boolean analogue of properties (1)-(9) in Theorem 5.1. It might be fruitful to consider the validity of property (6) in the Boolean and monotone cases in terms of the embeddings into tensor independence [11]. In free probability, most of the results of Theorem 5.1 are already known (see $[3,7]$ ) except for the failure of the free analog of property (6).

Another similarity between free and monotone independences is that the number of atoms in a $\boxplus$-infinitely divisible distribution is restricted in a similar way to the case of a $\triangleright$-infinitely divisible distribution (see Theorem 3.5 in [13] and Proposition 2.8 in [4]).

Acknowledgements. The author expresses his great appreciation to Professor Izumi Ojima for warm encouragement during his master course and for valuable discussions about independence and interacting Fock spaces. He thanks Professor Nobuaki Obata for giving him an opportunity to present this research in Tohoku University. He thanks Professor Naofumi 
Muraki for informing him about the reference [4. He also thanks Professor Uwe Franz for valuable discussions in Kyoto. He is also grateful to Mr. Hayato Saigo, Mr. Ryo Harada, Mr. Hiroshi Ando, and Mr. Kazuya Okamura for their interest in his research and many helpful discussions. This work was supported by JSPS (KAKENHI 21-5106).

\section{References}

[1] N. I. Akhiezer, The Classical Moment Problem, Oliver and Boyd, 1965.

[2] A. Alexiewicz et W. Orlicz, Remarque sur l'équation fonctionnelle $f(x+y)=f(x)+$ $f(y)$, Fund. Math. 33 (1945), 314-315.

[3] O. E. Barndorff-Nielsen and S. Thorbjørnsen, Classical and free infinite divisibility and Lévy processes, in: Quantum Independent Increment Processes II, Lecture Notes in Math. 1866, Springer, Berlin, 2006, 33-159.

[4] S. T. Belinschi, Complex analysis methods in noncommutative probability, $\mathrm{PhD}$ thesis, Indiana Univ., 2005; arXiv:math/0602343v1.

[5] F. Benaych-Georges, Taylor expansions of R-transforms, application to supports and moments, Indiana Univ. Math. J. 55 (2006), 465-481.

[6] H. Bercovici, Multiplicative monotonic convolution, Illinois J. Math. 49 (2005), 929951.

[7] H. Bercovici and V. Pata, Stable laws and domains of attraction in free probability theory (with an appendix by Philippe Biane), Ann. of Math. (2) 149 (1999), 10231060 .

[8] H. Bercovici and D. Voiculescu, Free convolution of measures with unbounded support, Indiana Univ. Math. J. 42 (1993), 733-773.

[9] E. Berkson and H. Porta, Semigroups of analytic functions and composition operators, Michigan Math. J. 25 (1978), 101-115.

[10] G. B. Folland, Real Analysis, Modern Techniques and Their Applications, 2nd ed., Wiley, 1999.

[11] U. Franz, Unification of boolean, monotone, anti-monotone, and tensor independence and Lévy processes, Math. Z. 243 (2003), 779-816.

[12] - Monotone and boolean convolutions for non-compactly supported probability measures, Indiana Univ. Math. J. 58 (2009), 1151-1186.

[13] T. Hasebe, Monotone convolution and monotone infinite divisibility from complex analytic viewpoint, Infin. Dim. Anal. Quantum Probab. Relat. Topics 13 (2010), 111-131.

[14] T. Hasebe and H. Saigo, The monotone cumulants, Ann. Inst. Henri Poincaré Probab. Statist., to appear; arXiv:0907.4896v3.

[15] N. Muraki, Monotonic independence, monotonic central limit theorem and monotonic law of small numbers, Infin. Dim. Anal. Quantum Probab. Relat. Topics 4 (2001), 39-58.

[16] —, Monotonic convolution and monotonic Lévy-Hinčin formula, preprint, 2000.

[17] K. Sato, Lévy Processes and Infinitely Divisible Distributions, Cambridge Univ. Press, Cambridge, 1999.

[18] J. M. Shapiro, A condition for existence of moments of infinitely divisible distributions, Canad. J. Math. 8 (1956), 69-71. 
[19] R. Speicher and R. Woroudi, Boolean convolution, in: Free Probability Theory, D. Voiculescu (ed.), Fields Inst. Comm. 12, Amer. Math. Soc., 1997, 267-280.

Takahiro Hasebe

Graduate School of Science

Kyoto University

Kyoto 606-8502, Japan

E-mail: hsb@kurims.kyoto-u.ac.jp

Received February 16, 2010

Revised version July 23, 2010 